\title{
Multipartite entanglement generation and fidelity decay in disordered qubit systems
}

\author{
Simone Montangero 1 , $*$ and Lorenza Viola ${ }^{2}$ \\ ${ }^{1}$ NEST-CNR-INFM \& Scuola Normale Superiore, Piazza dei Cavalieri 7, 56126 Pisa, Italy \\ ${ }^{2}$ Department of Physics and Astronomy, Dartmouth College, 6127 Wilder Laboratory, Hanover, NH 03755, USA
}

(Dated: November 13, 2018)

\begin{abstract}
We investigate multipartite entanglement dynamics in disordered spin- $1 / 2$ lattice models exhibiting a transition from integrability to quantum chaos. Borrowing from the generalized entanglement framework, we construct measures for correlations relative to arbitrary local and bi-local spin observables, and show how they naturally signal the crossover between distinct dynamical regimes. Analytical estimates are obtained in the short- and long-time limits. Our results are in qualitative agreement with predictions from random matrix theory and relevant to both condensed-matter physics and to the stability of quantum information in disordered quantum computing hardware.
\end{abstract}

PACS numbers: 03.67.Mn, 03.67.Lx, 05.45.Mt, 24.10.Cn

Developing a quantitative understanding of the structural and dynamical properties of entanglement in manybody quantum systems is a critical challenge for both condensed-matter theory and quantum information science. Low-dimensional disordered spin models offer, in this context, an ideal testbed for theoretical analysis. On one hand, these systems are simple enough for analytic benchmarks to exist in limiting situations, yet capable to demonstrate a broad typology of complex quantum phenomena. The latter range from field- or disorder-driven structural ground-state changes responsible for quantum phase transitions [1], to dynamical crossovers from integrable to non-integrable regimes and the emergence of quantum chaos [2]. On the other hand, arrays of interacting spin- $1 / 2$ s naturally schematize quantum computing hardware as diverse as semiconductor quantum dots [3], superconducting Josephson qubits [4], electron floating on He [5], and optical lattices [ $[\underline{6}$ - disorder resulting from the unavoidable presence of imperfections in the singlequbit energy spacings and/or inter-qubit couplings.

Following 7], intense effort has been devoted to both assess the impact of disorder on quantum computing performance [8, 9, 10], and to characterize entanglement across the ensuing transition to quantum chaos 11, 12]. These studies point to the key role of spectral properties, as captured by the Local Density of States (LDOS) 13], in determining the system stability against the disorder. Changes in the LDOS profile are ultimately responsible for the existence of different dynamical regimes, as reflected by qualitative changes in the rate of fidelity decay 14]. Numerical experiments confirm that the same regimes translate into distinctive static 12 and dynamic 15] properties of pairwise entanglement, as quantified by concurrence [16]. While providing suggestive evidence, such analyses are not fully satisfactory for two reasons. First, concurrence lacks a direct physical interpretation, hindering the possibility to relate entanglement to spectral properties. Second, concurrence is a bipartite measure, preventing the quantification of multipartite correlations which dominate in strongly coupled scenarios.
In this Letter, we overcome the above limitations by exploiting Generalized Entanglement (GE) [17] as a setting for defining entanglement relative to arbitrary sets of observables. In [18, GE measures constructed from algebras of fermionic operators have been applied to the study of broken-symmetry quantum phase transitions in exactly solvable models, notably the spin- $1 / 2 \mathrm{XY}$ chain in a transverse field. Here, we show how GE contributes to the understanding of standard multipartite correlations between distinguishable systems, by selecting local and bi-local algebras of observables corresponding to single and pairs of qubits, respectively. Beside providing a transparent interpretation of the results based on concurrence 12, 15], our approach allows for quantitative insight about entanglement dynamics starting from arbitrarily correlated initial states. We quantify the influence of LDOS properties in two limits: at short-time, by establishing a direct link with fidelity decay; at long times, by relating the entanglement saturation value to the participation ratio of the asymptotic many-body state.

The model.- We focus on a two-dimensional lattice of $n$ disordered spin- $1 / 2$ particles ( $n$ even) described, in units $\hbar=1$, by the following Hamiltonian:

$H=\sum_{j=1}^{n}\left[\Delta+\delta_{j}\right] \sigma_{z}^{(j)}+\sum_{\langle i, j\rangle} J_{i j} \sigma_{x}^{(i)} \sigma_{x}^{(j)} \equiv H_{\Delta}+H_{\delta}+H_{J}$,

where $\sigma_{\alpha}^{(i)}, \alpha \in\{0, x, y, z\}, \sigma_{0}^{(i)}=\mathbb{I}$, are Pauli operators, and $\langle i, j\rangle$ stands for nearest-neighbor sites. Open boundary conditions and energy units $\Delta \equiv 1$ are used. The parameters $\delta_{j}, J_{i j}$ characterize the disorder in the on-site energy splittings and two-body couplings, respectively. We assume that $\delta_{j}, J_{i j}$ are uniformly random over $[-\delta, \delta]$, $[-J, J]$, with $\delta, J>0$. The above model, which belongs to the random transverse-field Ising lattice class [19], was recently used to describe a quantum register with static imperfections [7]. Understanding the influence of the latter may be especially important for controlling entanglement in quantum computers with always-on interactions 20] and one-way quantum architectures 21]. 


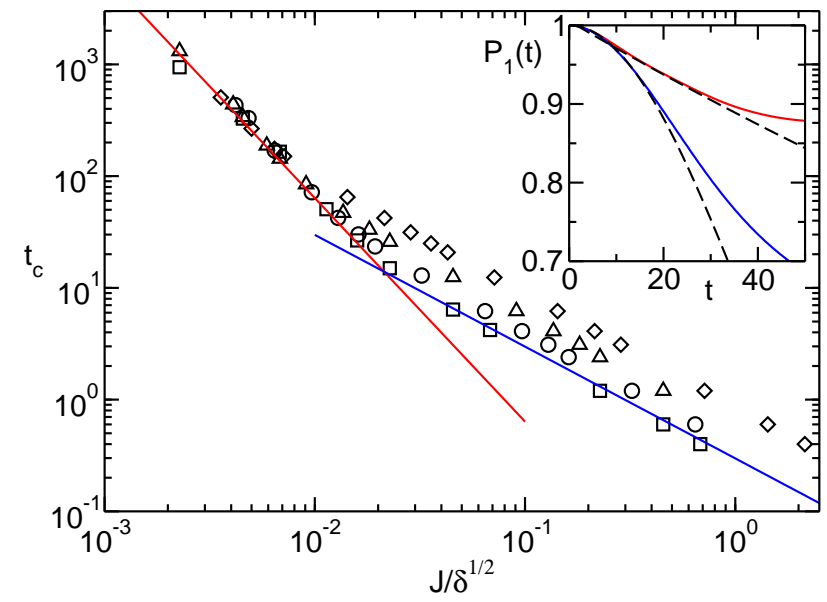

FIG. 1: (Color Online) Local purity critical time $t_{c}\left(P_{1}\left(t_{c}\right)=\right.$ 0.9 ) as a function of $J / \delta$ for $\delta=0.02,0.05,0.1,0.2$ (diamonds, triangles, circles, squares). To smooth statistical fluctuations, here and in the following we consider averages $\left\langle P_{1}(t)\right\rangle_{D}$ over a number $N_{r}=10$ of disorder realizations. We assume $n=10$ unless otherwise specified. Inset: Time evolution of $P_{1}(t)$ in the FGR $J=\delta / 10=0.01$ (red) and ergodic $J=\delta=$ 0.1 (blue) regime starting from the state of Eq. (5) for $n=$ $14, n_{B}=0$. Dotted lines: Exponential and Gaussian fits, as in Eq. (3).

The spectrum of $H_{\Delta}$ is composed of $n+1$ levels, with energy $E_{B}=\Delta(2 k-n)$ and degeneracy $N_{B}(k)=$ $n ! /[k !(n-k) !], k=0, \ldots, n$. When $\delta, J \ll \Delta$, the degeneration is removed and $n+1$ bands appears. The width $\Delta_{B}$ of the bands depends on the ratio $J / \delta[7]$. In the limit where $J \ll \delta, \Delta_{B} \sim \delta \sqrt{n}$ as the spread is led by the diagonal term $H_{\delta}$. Correspondingly, bands are Gaussian. Inside each band, the effective number of states coupled by $H_{J}$ is given by the width of the LDOS. For $J \lesssim \delta / n \equiv J_{c}$, $H_{J}$ couples few states, and the system is slightly perturbed, while for $J>J_{c}$ quantum chaos sets in and the unperturbed levels are coupled to a quasi-continuum set of states. The LDOS becomes Lorentzian, with a width determined by the Fermi Golden Rule (FGR), $\Gamma_{F}=J^{2} n / \delta$ [7]. Increasing $J$, when $\Gamma_{F} \sim \Delta_{B}$, that is for $J \sim J_{E}=\delta / n^{1 / 4}$, all the levels inside a given band are mixed and the LDOS approaches the level density. Thus, the LDOS becomes a Gaussian with width $\Gamma_{E} \sim J$ [7]. In summary, three distinct regimes exist - perturbative $\left(J<J_{c}\right)$, FGR $\left(J<J_{E}\right)$, and ergodic $\left(J_{E}<J<\Delta\right)$. It has been shown that starting from an eigenstate $\left|\psi_{0}\right\rangle$ of $H_{\Delta}$, the survival probability (fidelity henceforth, as for initial eigenstates the two quantities coincide) $F(t)=\left|\left\langle\psi_{0} \mid \psi_{t}\right\rangle\right|^{2}$ also follows three different behaviors: $F(t)$ oscillates near one in the perturbative regime, while it decays as an exponential or a Gaussian in the FGR and ergodic regime, respectively []]. The connection between LDOS shape and fidelity decay has been unveiled in [8] by means of a perturbative relation between $F(t)$ and the Fourier transform of the LDOS.
Local and bi-local purities.- An initial pure state $\left|\psi_{0}\right\rangle$ evolving under $H$ remains pure for any fixed disorder realization. Accordingly, we consider pure-state entanglement throughout. The basic intuition underlying the GE notion [17] is to quantify how entangled a state $|\psi\rangle$ is relative to an observable set $\mathcal{O}$ in terms of how pure $|\psi\rangle$ remains upon restricting operational access to $\mathcal{O}$. While no single set $\mathcal{O}$ can exhaust the complexity of multipartite entanglement, two simple choices will illustrate the usefulness and flexibility of the algebraic approach. First, we consider the set $\mathcal{O}=\mathcal{O}_{1}$ generated by arbitrary local observables that is, we probe the average entanglement of each qubit with the rest of the lattice. The corresponding (time-evolved) local purity measure is defined as

$$
P_{1}\left(\left|\psi_{t}\right\rangle\right)=\frac{1}{n} \sum_{\alpha=x, y, z}^{i=1, n}\left|\left\langle\psi_{t}\left|\sigma_{\alpha}^{(i)}\right| \psi_{t}\right\rangle\right|^{2} .
$$

For this choice of observables, GE coincides with global multipartite entanglement as quantified by the MeyerWallach metric $Q$ [18, 22], with $Q=1-P_{1}$ [18, 23]. As a second observable set, whose physical motivation will become clear later, we choose $\mathcal{O}=\mathcal{O}_{2}$ generated by all the observables acting on pairs of nearest neighbor spins. We compute a bi-local purity measure as

$$
P_{2}\left(\left|\psi_{t}\right\rangle\right)=\frac{2}{3 n}{\widetilde{\sum_{\alpha, \beta=x, y, z, 0}}}_{i, j\rangle}\left|\left\langle\psi_{t}\left|\sigma_{\alpha}^{(i)} \sigma_{\beta}^{(j)}\right| \psi_{t}\right\rangle\right|^{2}
$$

where the tilde means that the identity term with $\alpha=$ $\beta=0$ is omitted. $P_{2}$ may be thought as a "coarsegrained" version of $P_{1}$, resulting from ignoring the fine structure given by arbitrary correlations within each pair. Both measures are normalized to give one (zero) on states which are fully separable (contain maximal GE) relative to the corresponding algebra. Remarkably, both $P_{1}$ and $P_{2}$ are directly measurable quantities in principle [23].

Initial separable state.- We first study multipartite entanglement generation starting from a separable state in the central band $(k=n / 2)$ that is, $\left|\psi_{0}\right\rangle=$ $|010101 \ldots 01\rangle \equiv|c\rangle$, where $\{0,1\}$ label the states of each spin in the computational basis and $c$ is the integer given by the corresponding binary string. The evolution of $P_{1}(t)$ under different values of $J$ at fixed $\delta$ are depicted in the inset of Fig. 1 The decay of $P_{1}(t)$ clearly follows two different dynamical laws. Specifically, data in the FGR and in the ergodic regime are described, respectively, by

$$
P_{1}^{F}(t) \approx e^{-C \Gamma_{F} t}, \quad P_{1}^{E}(t) \approx e^{-C^{\prime} \Gamma_{E}^{2} t^{2}}
$$

where the constants $C, C^{\prime}$ depend on the initial state and the lattice coordination number (see e.g. 24]). Let $t_{c}$ be the time it takes for $P_{1}$ (or $P_{2}$ ) to reach the value $K$ e.g., $P_{1}\left(t_{c}\right)=K$ : We find that $t_{F}^{c} \sim 1 / J^{2}$ in the FGR regime, whereas $t_{E}^{c} \sim 1 / J$. These behaviors have been verified over a wide range of disorder parameters, see Fig. [1 


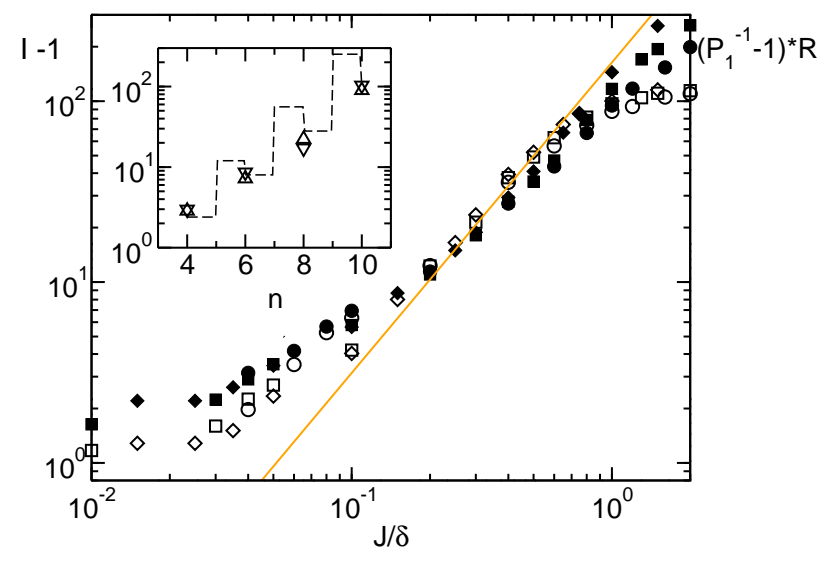

FIG. 2: (Color Online) Saturation values of I-1 (empty symbols) and of $\left(P_{1}^{-1}-1\right) \cdot R$ (filled symbols) vs $J / \delta$ for $\delta=0.05$ (circles), $\delta=0.1$ (squares), $\delta=0.2$ (diamonds), and rescaling factor $R=7$. The straight line is proportional to $C(J / \delta)^{2} N_{B}(k=n / 2), C \sim 0.7$. Inset: Saturation value of the IPR (triangles up) and $P_{1}^{-1}$ (triangles down) in the ergodic regime $(\delta=0, J=0.1)$ as a function of $n$. The dashed line gives $N_{B}(n / 2)$ vs $n$.

A simple physical interpretation of the above results follows from the possibility to directly relate GE dynamics to fidelity decay for any computational eigenstate $|c\rangle$. While a full derivation will be presented elsewhere, the key steps are (i) to realize that only $\sigma_{z}^{(i)}$ observables contribute to the evolution of $P_{1}(t)$ (ii) to isolate the fidelity term $\left|\left\langle c\left|e^{-i H t}\right| c\right\rangle\right|^{2}$ in the resulting $z$-purity. This yields

$$
P_{1}(t)=F(t)^{2}+\frac{1}{n} \sum_{j=1}^{n}\left(2 F(t)(-)^{\pi_{j}(c)} \alpha_{j}(t)+\alpha_{j}(t)^{2}\right),
$$

where $\alpha_{j}(t)=\sum_{p \neq c}\left|\left\langle p\left|e^{-i H t}\right| c\right\rangle\right|^{2}(-)^{\pi_{j}(p)}$ and $(-)^{\pi_{j}(q)}$ $=(-)^{\left\lfloor q / 2^{j-1}\right\rfloor}$ is the parity of the $j$ th qubit in the computational state $|q\rangle$. One can show that each term $\alpha_{j}(t)$ is of order $\mathcal{O}\left((J / \delta)^{2} t^{2}\right)$. Thus, according to the above equation, the connection between local purity and fidelity decay (hence LDOS via the relation found in [8]) becomes exact in the limit $t \rightarrow 0$. For sufficiently short times, the first term still dominates and the dynamics is governed by $F(t)^{2}$, whereby the two regimes of Eq. (3) arise.

For times much longer than $\Gamma_{E, F}$, the asymptotic GE amount may be estimated using random matrix theory [2]. By working within the Gaussian orthogonal ensemble, we represent the many-body state $\left|\psi_{\infty}\right\rangle$ as a random superposition of $N_{\infty}$ unperturbed states within the central band that is, $\left|\psi_{\infty}\right\rangle=$ $\sum_{p=1}^{N_{\infty}} w_{p}|p\rangle$, with amplitudes $w_{p}$ satisfying the normalization constraint. For sufficiently large $N_{\infty}$, correlations between different components may be neglected, and the probability density of each component is well approximated by the Porter-Thomas distribution, $P_{\mathrm{PT}}\left(\left|w_{p}\right|^{2}\right)=\left(2 \pi N_{\infty}\left|w_{p}\right|^{2}\right)^{-1 / 2} \exp \left(-N_{\infty}\left|w_{p}\right|^{2} / 2\right)$. Evaluating the disorder-averaged asymptotic local purity

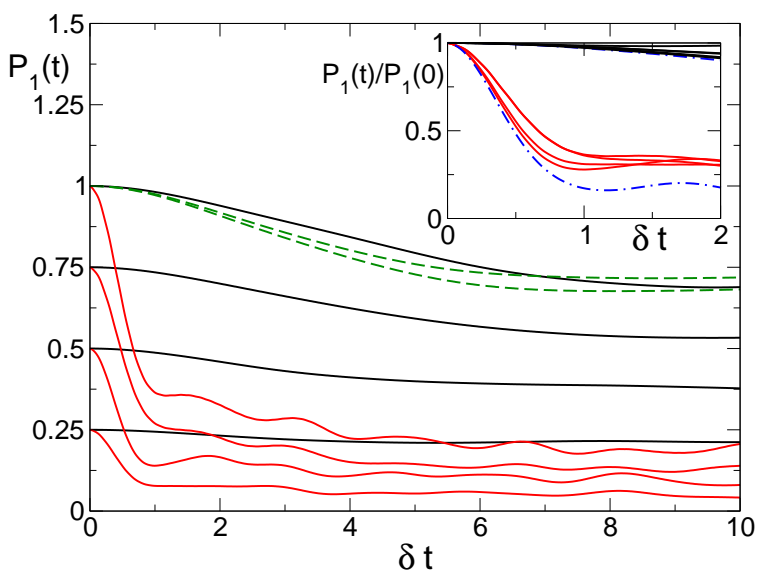

FIG. 3: (Color Online) Local purity $P_{1}(t)$ versus time in the FGR (black lines) and ergodic regime (red lines) for different initial states with $n_{B}=0,1,2,3, \delta=0.1$, and $J=0.01$ (black curves), $J=0.1$ (red curves). Dashed lines: Different separable initial states $\left|\psi_{0}\right\rangle=|0101010110\rangle,|010101010\rangle \otimes$ $(|0\rangle+|1\rangle) / \sqrt{2}$ for $\delta=0.1, J=0.01$. Inset: The sames curves rescaled by $P_{1}(0)$. Also shown (dot-dashed lines) is the local purity decay in the ergodic and FGR regimes for an initial $|W\rangle$ state.

yields $\left\langle P_{1}^{\infty}\right\rangle_{D}=\left\langle P_{1}^{\infty}\right\rangle_{\mathrm{PT}}=2 / N_{\infty}$, consistent with the scaling $3 /\left(N_{\infty}+1\right)$ predicted for a random pure state under the invariant Haar measure [25].

Let the degree of delocalization of $\left|\psi_{\infty}\right\rangle$ be quantified by the Inverse Participation Ratio (IPR) 2, 7, 12] that is, $\mathrm{I}=1 / \xi$, where the asymptotic participartion number $\xi^{\infty}=\sum_{p}\left|w_{p}\right|^{4}$. By estimating $\left\langle\xi^{\infty}\right\rangle_{D}=3 / N_{\infty}$, $\left\langle P_{1}^{\infty}\right\rangle_{D}=2 / 3\left\langle\xi^{\infty}\right\rangle_{D}$ - thus, GE directly quantifies delocalization in this regime. Similarly, $\left\langle\mathrm{I}^{\infty}\right\rangle_{D} \approx N_{\infty} / 3$, up to deviations of the order $\mathcal{O}\left(N_{\infty}^{-1 / 2}\right)$, consistent with Gaussian fluctuation statistics [2]. Because the dynamically accessible states are determined by the ratio between the LDOS width and the bandwidth, we further estimate $N_{\infty} \approx\left(\Gamma_{F} / \Delta_{B}\right) N_{B}$ for any initial eigenstate in the central band and in the FGR regime, whereas the IPR is constant in the ergodic regime. These predictions have been confirmed numerically (see Fig. 2) 26]. Thus,

$$
\left\langle P_{1}^{\infty}\right\rangle_{D} \propto \frac{1}{\left\langle\mathrm{I}^{\infty}\right\rangle_{D}} \propto \frac{\Delta_{B}}{\Gamma_{F} N_{B}}
$$

As shown in Fig. 2. Eq. (44) qualitatively agrees with data over an extensive range of parameters. Note that these asymptotic results, as well as the initial decay laws of Eq. (3), are valid for the dynamics of any initial separable state, not just of a computational state (see Fig. 3).

Initial entangled states.- The analysis may be extended to the dynamics of arbitrarily entangled initial states. Consider a state in the central band containing only bi- 
partite entanglement first, for instance

$$
\left|\psi_{0}\left(n_{B}\right)\right\rangle=\overbrace{|01 \ldots 01\rangle}^{n-2 n_{B}} \otimes\left[\frac{1}{\sqrt{2}}(|01\rangle+|10\rangle)\right]^{\otimes n_{B}},
$$

where $n_{B}$ is the number of Bell pairs, $n_{B}=0$ recovering the fully separable case. The local purity evolution is depicted in Fig. 3. Reflecting the fact that $P_{1}$ is sensitive to all correlations between spins, the initial value is lower the larger $n_{B}, P_{1}(0)=1-2 n_{B} / n$, using Eq. (11). For $t>0, P_{1}(t)$ decays similarly to the separable case, two distinct dynamical regimes emerging for $n_{B}=0,1,2,3$. As expected, data corresponding to a given regime approximately fall on the same curve once rescaled by $P_{1}(0)$ (Fig. [3 inset). This may be quantitatively understood by studying the evolution of the bi-local purity defined in Eq. (2). By construction, $P_{2}$ is insensitive to any pairwise correlation present in the state (5), effectively mapping the analysis back to the separable case $n_{B}=0$.

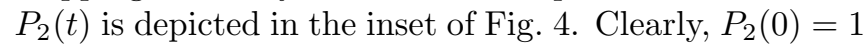
irrespective of $n_{B}$. The time decay then follows the two regimes predicted by (3), again reflecting the underlying structure of the LDOS (see Fig. (4).

As a representative example of an initial state containing genuine multipartite entanglement, we focus on a so-called $|W\rangle$ state that is, an equally weighted superposition with equal phases of the $N_{B}(1)=n$ states with $z$-magnetization $\sum_{j} \sigma_{z}^{(j)}=n-2$. Within our disorder model, $|\mathrm{W}\rangle$ is automatically protected against the effects of $H_{\delta}, H_{J}$ as long as the coupling between different bands remains small, as assumed so far. Indeed, the local purity remains almost constant (data not shown). Thus, to analyze how the (tripartite) correlations contained in a $|\mathrm{W}\rangle$ are affected by static random imperfections we set $\Delta=0$ 27. Again, the same qualitative picture arises, with two distinct dynamical regimes (inset of Fig. (3) and two different scalings of critical decay times $t_{c}$ (Fig. 4).

Conclusions.- We established the existence of distinctive signatures in the dynamics of multipartite entanglement in a quantum many-body system subject to static disorder. While a more systematic analysis is certainly desirable, we expect our main conclusions to prove valid under more general conditions, including different dimensionality and/or model Hamiltonians, as well as higherspin systems. Beside reinforcing the usefulness of the GE notion as a diagnostic framework for complex quantum systems, the deep connections between GE, LDOS, and fidelity decay emerging from our work are likely to have broader implications across the fields of quantum information, condensed-matter physics, and quantum chaos, stimulating crosstalk between different communities.

We thank W. G. Brown, R. Fazio, G. Ortiz, L. F. Santos, and Y. S. Weinstein for feedback. Partial support from the IST-SQUBIT2, from IBM (Faculty Awards 2005), and from Constance and Walter Burke through their Special Projects Fund in QIS is acknowledged.

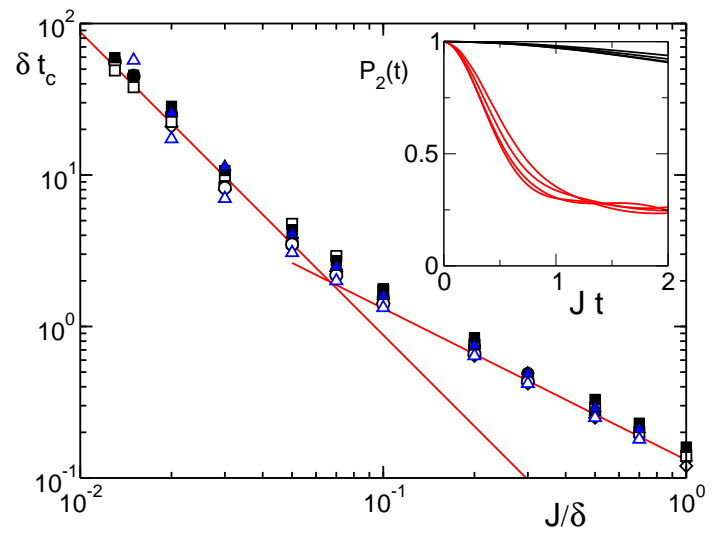

FIG. 4: (Color Online) Purity critical time $t_{c}$ as a function of $J / \delta$ for different initial states, $n_{B}=1$ (circles), $n_{B}=2$ (squares), $n_{B}=3$ (diamonds), $|\mathrm{W}\rangle$ (blue triangles,) and different purity measures: $P_{1}(t)$ (empty) and $P_{2}(t)$ (full). Inset: $P_{2}(t)$ vs time in the FGR regime (black lines) and in the ergodic regime (red lines) for different initial states with $n_{B}=0,1,2,3$.

* Corresponding author. E-mail: monta@sns.it

[1] S. Sachdev, Quantum Phase Transitions (Cambridge University Press, Cambridge, UK, 1999).

[2] F. Haake, Quantum Signatures of Chaos (Springer, Berlin, 1991).

[3] V. Cerletti et al, Nanotechnology 16, R27 (2005).

[4] Y. Makhlin, G. Schoen, and A. Shnirman, Rev. Mod. Phys. 73, 357 (2001).

[5] M. I. Dykman et al, Quant. Inf. Comput. 5, 335 (2005).

[6] A. Kay and J. K. Pachos, New J. Phys. 6126 (2004).

[7] B. Georgeot and D. L. Shepelyansky, Phys. Rev. E 62, 3504 (2000); ibid. 62, 6366 (2000).

[8] V. V. Flambaum, Aust. J. Phys. 53, 489 (2000).

[9] G. Benenti et al, Phys. Rev. Lett. 87, 227901 (2001).

[10] G. P. Berman et al, Phys. Rev. E 64, 056226 (2001).

[11] L. F. Santos, G. Rigolin, and C. O. Escobar, Phys. Rev. A 69, 042304 (2004).

[12] C. Mejia-Monasterio et al, Phys. Rev. A 71, 062324 (2005).

[13] The LDOS $\rho_{w}$ describes the profile $\left|W_{i}\right\rangle$ of an eigenstate of an unperturbed quantum system over the eigenbasis $\left\{\left|\tilde{W}_{j}\right\rangle\right\}$ of the perturbed version (see e.g. [7]): $\rho_{w}(E-$ $\left.E_{i}\right)=\sum_{j}\left|\left\langle W_{i} \mid \tilde{W}_{j}\right\rangle\right|^{2} \delta\left(E-E_{j}\right)$.

[14] See e.g. G. Benenti and G. Casati, Phys. Rev. E 65, 066205 (2002), T. Prosen, Th. H. Seligman, and M. Znidaric, Progr. Theor. Phys. Suppl. 150, 200 (2003).

[15] S. Montangero, G. Benenti, and R. Fazio, Phys. Rev. Lett. 91, 187901 (2003).

[16] W. Wootters, Phys. Rev. Lett. 80, 2245 (1998).

[17] H. Barnum et al, Phys. Rev. A 68, 032308 (2003); H. Barnum et al, Phys. Rev. Lett. 92, 107902 (2004).

[18] R. Somma et al, Phys. Rev. A 70, 042311 (2004).

[19] I. M. Lifshits, S. A. Gradeskul, and L. A. Pastur, Introduction to the Theory of Disordered Systems (Wiley, New York, 1988).

[20] S.C. Benjamin and S. Bose, Phys. Rev. Lett. 90, 247901 (2003). 
[21] R. Raussendorf and H.-J. Briegel, Phys. Rev. Lett. 86, 5188 (2001).

[22] D. Meyer and N. R. Wallach, J. Math. Phys. 43, 4273 (2002).

[23] G. K. Brennen, Quantum Inf. Comput. 3, 619 (2003).

[24] P. Facchi et al, Phys. Rev. A 71, 060306(R) (2005).
[25] A. J. Scott and C. M. Caves, J. Phys. A 36, 9553 (2003).

[26] Interestingly, a similar dependence upon $(J / \delta)^{2}$ has been found for the saturation value of $F(t)$ in Y. S. Weinstein et al, Quant. Inf. Proc. 1, 439 (2003).

[27] We could alternatively choose $J>\Delta$. 